\title{
Combined Analyses Procedure of Failure Modes and Risk Phenomena Using the Concept of Normal State Conditions
}

\author{
Yuichi Otsuka*, Takeshi Ishizaki, Yukio Miyashita, Yoshiharu Mutoh \\ Top Runner Incubation Center for Academia-Industry Fusion, Nagaoka University of Technology, \\ Nagaoka, Japan \\ Email: *otsuka@vos.nagaokaut.ac.jp
}

Received August 1, 2012; revised September 5, 2012; accepted September 20, 2012

\begin{abstract}
Normal failure or risk analyses procedure contains the following steps: 1) modeling process, 2) assessment process of its hazardous extent. 1) Modeling process is the considering procedure that sets the sequence of happening failures or risks. 2) Assessment process is the evaluation procedure that express its hazardous extent quantitatively or qualitatively (probability, seriousness of injury etc.). According to ISO14120 (Risk assessment process), ranking procedure of risks are established. However, there is no logical procedure for 1. Modeling process and these steps still highly depends on designer's knowledge or experiences of failures and accidents. It is necessary to establish a logical guideline of failure modeling process for fresh designers in order to effectively conduct failure and risk analyses with their acceptable workloads. This study aims at proposing the logical failure modeling process based on the SSM (Stress-strength model) and the normal-state conditions. In the beginning, designers make a stress-strength model of considering components and its "normal condition". Introducing "deviations" in normal conditions of stress-strength state and surrounding environmental conditions can lead the designers to easily predict failure modes caused by the proposed deviations. Similar steps are applied in the process of considering risk phenomena caused by failure modes. A case study of assessing the safety of micro windmill demonstrated the effectiveness of our proposed procedures.
\end{abstract}

Keywords: Failure Modes and Effects Analyses (FMEA); Risk Assessment; Design Review; System Safety

\section{Introduction}

Normal flows of failure modes and risk analyses are composed by the two steps: 1) modeling process is setting hazard and failure scenario, 2) assessment process is a quantification of probability of failures or risks and its degree of damages for systems or humans [1,2]. ISO safety code 14120 and another researches proposed the assessment processes both quantitative and qualitative categorizing methods. However, the modeling process, which is considered critical for valid assessment processes, have widely been based on practitioner's experiences. If the practitioner has insufficient knowledge, which will cause the insufficient quality of failure analyses because he or she can predict little failure modes. Even if the cases of veterans, they tend to focus on their experiences and then another failure modes are sometimes omitted. Both cases cannot produce sufficient result of failure analyses. Therefore, logical modeling guidelines to predict sufficient failure modes are indispensable.

\footnotetext{
"Corresponding author.
}

This paper proposes a logical modeling procedure for failure modes by combining SSM (Stress-Strength Model) and patterning of deviations from normal states of SSM in order to predict failure modes caused by the deviations [3-7]. The proposed process is applied to assess the failure modes of windmill system. The authors then call the proposed model combined failure-risk prediction model.

\section{Combined Failure-Risk Prediction Model from Normal States of SSM}

Figure 1 shows the entire flowchart of FMEA and risk assessment. The process is composed of the following steps.

\subsection{Specifying Target Systems}

1) Decide components; mechanical or electrical components of the target system should be specified at first. Summary of component and structural links are expressed as shown in Figure 2. 


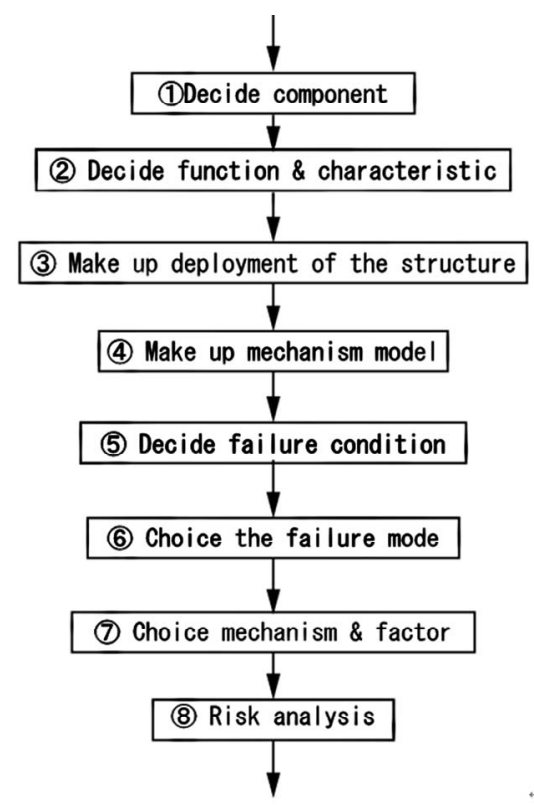

Figure 1. Flow chart of FMEA and risk assessment.

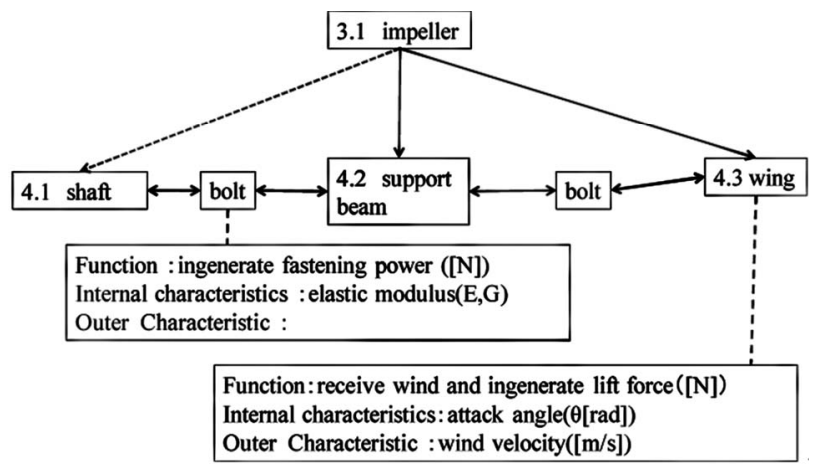

Figure 2. Function deployment diagram of a micro windmill. Link model is defined to show the connections among elements.

2) Decide function and characteristics; functions for each element should be specified such as function names, dimensions of parameters, a definition range of parameters, connecting conditions to neighbor elements in order to set normal conditions of the element. Design characteristics, which is necessary to illustrate the normal functional states of the elements, are then identified. The design characteristics involves two components; internal one which is material properties or dimensions of the components etc. and outer one which is defined by environmental conditions such as wind speed.

3) Making functional deployment diagram (Figure 3); individual components which were defined in the previous step should be interconnected to construct structures. Joining link and components are simplified as shown in Figure 3.

4) Making mechanism models (Figure 3); all system have energy flow from input sources to output target in order to work something designed. The example of a windmill is shown in Figure 3. To transform kinetics energy of wind to electricity, the components in the windmill successfully connects with each other.

\subsection{Failure Modes Effects Analyses}

1) Choice of failure modes; in conventional method, setting failure modes is highly depending on experiences of practitioners [4,5]. Figure 4 shows the logical procedure of identifications of failure modes from the normal state of functions shown in Figure 3. Introducing deviation patterns (plus, minus or inserting another conditions and deteriorating necessary conditions) into the normal state can determine the abnormal modes of the functional model. In this process, guide-word list as shown in Table $\mathbf{1}$ is helpful to set sufficient patterns of deviations. Table 1 includes a plenty of failure modes according to excessive loading (plus conditions) or insufficient minus conditions. For instance the column "tensile", which is connecting to plastic deformations or cracking of structural components, unusual higher value $(+)$ of tensile loading can lead to "crack" and unusual continuous loading "f+" can also result in "creep" damage mode, respectively. In the case of the column "heat", +conditions and -conditions can reach different unusual state such as "melting" or "solidifications". In specifying some abnormal modes, at first $+/$ - conditions

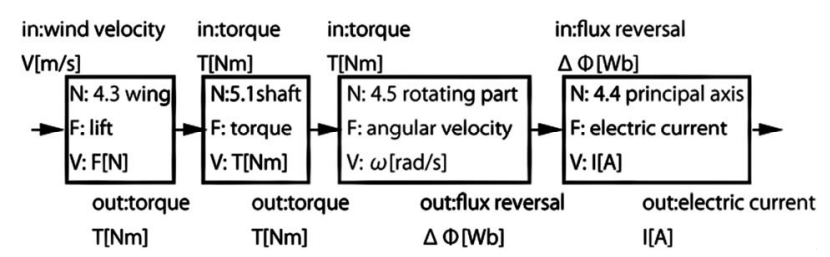

Figure 3. Functional block diagram of the windmill.

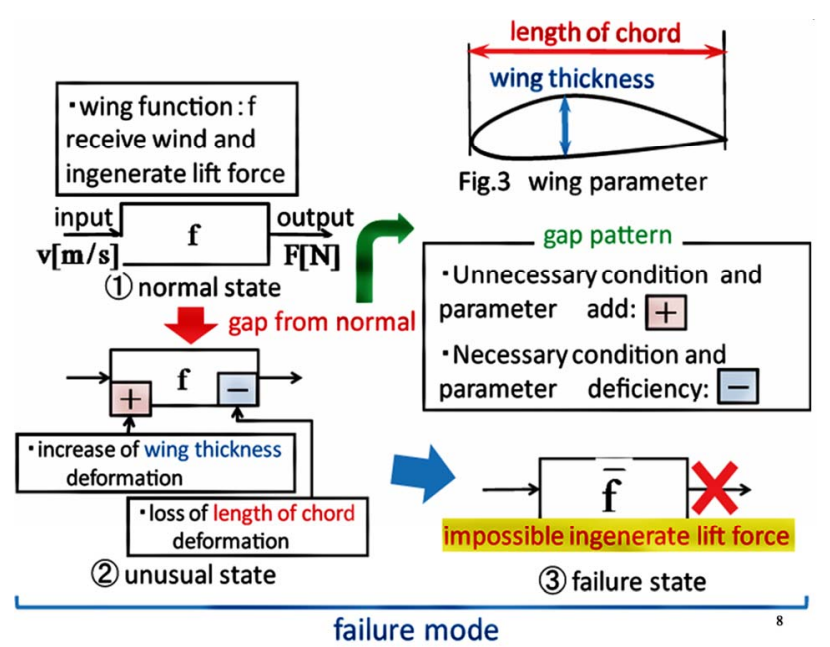

Figure 4. Concept of failure mode analyses by patterning deviations from normal states of SSM. 
on normal state (Figure 3) are set. By searching same deviations on Table 1, abnormal mode relating to the introduced deviation can easily be determined. Failure modes are finally named by combinations of abnormal modes and functional failure, such as "Impossible in generating lift force due to deformation".

2) Choice mechanism or causes; in order to determine solutions for failure modes, mechanism of the failure modes and its cause should be analyzed. In current study, mechanisms of failure modes in Stress-Strength model (SSM) are summarized two forms; excessive loading $(+)$ or insufficient strength $(-)$, respectively. This mechanism could be prevented by treating during design or manu- facturing, maintenance stages. Therefore cause of mechanisms are design, manufacturing or operating failures etc. In analyzing failure modes, fault tree analyses (FTA) is much effective.

3) Risk assessment; in the case of hazard modeling process, similar process is applicable. At first, normal state of service is determined. By using the deviations patterns, predictable error scenario and hazardous conditions by failure modes are determined.

Table 2 shows the result of FMEA by using the proposed method. The proposed model will be applied in the phase of conceptual design and manufacturing design stage, which is the earlier periods of product developments.

Table 1. Guide word sheet of failure modes prediction.

\begin{tabular}{|c|c|c|c|c|c|}
\hline element & & $(+)$ & $\int \mathrm{d} t s s(+)$ & $(-)$ & $\int \mathrm{d} t(-)$ \\
\hline \multirow{3}{*}{ static loading } & \multirow{3}{*}{$\begin{array}{c}\text { tensile } \\
\text { compression } \\
\text { torsion }\end{array}$} & crack & creep & & \\
\hline & & buckling & creep & & \\
\hline & & crack & creep & & \\
\hline \multirow{3}{*}{$\begin{array}{l}\text { dynamic } \\
\text { loading }\end{array}$} & \multirow{3}{*}{ repeated-loading } & crack & fatigue & & \\
\hline & & buckling & setting & & \\
\hline & & crack & fatigue & & \\
\hline \multirow{3}{*}{ environment } & heat & melting & deterioration & solidification & deterioration \\
\hline & humidity(water) & wet/swellheaded & set/swellheaded & dry/deterioration & dry/deterioration \\
\hline & irruption of some thing & $\begin{array}{l}\text { break or deformation with } \\
\text { crackup }\end{array}$ & pollution & $\begin{array}{l}\text { internal structural } \\
\text { change }\end{array}$ & $\begin{array}{l}\text { internal structural } \\
\text { change }\end{array}$ \\
\hline \multirow[t]{3}{*}{ resistance } & cutting work & deformation/break & short measurement & $\begin{array}{l}\text { excessive } \\
\text { measurement }\end{array}$ & $\begin{array}{l}\text { excessive } \\
\text { measurement }\end{array}$ \\
\hline & deformation & break & & & \\
\hline & structure/shape & backlash & & rigid & \\
\hline
\end{tabular}

Table 2. FMEA data sheet of the windmill.

\begin{tabular}{|c|c|c|c|c|c|c|}
\hline \multirow{2}{*}{ class 4} & \multirow{2}{*}{ access point } & \multirow{2}{*}{$\begin{array}{l}\text { name of } \\
\text { element }\end{array}$} & \multirow{2}{*}{ function } & \multirow{2}{*}{$\begin{array}{l}\text { value of } \\
\text { valiable }\end{array}$} & \multicolumn{2}{|c|}{ failure mode } \\
\hline & & & & & add or deficiency & failure condition \\
\hline \multirow[b]{2}{*}{4.1} & & \multirow[b]{2}{*}{ shaft (3.1) } & \multirow[b]{2}{*}{ transmit torque } & \multirow[b]{2}{*}{$\mathrm{y}[\mathrm{m}]$} & 5.1 & \multirow[b]{2}{*}{ impossible to transmit } \\
\hline & & & & & 5.2 & \\
\hline \multirow{3}{*}{4.2} & \multirow{3}{*}{$4.3,5.2,5.3$ (bolt) } & \multirow{3}{*}{$\begin{array}{c}\text { support } \\
\text { beam }(3.1)\end{array}$} & \multirow{3}{*}{$\begin{array}{l}\text { fix radial derection of } \\
\text { wing }\end{array}$} & \multirow{3}{*}{$\mathrm{r}[\mathrm{m}]$} & bending deformation & \\
\hline & & & & & fracture & impossible to fix \\
\hline & & & & & 6.3 & \\
\hline \multirow{2}{*}{4.3} & \multirow{2}{*}{4.2 (bolt) } & \multirow{2}{*}{ wing (3.1) } & \multirow{2}{*}{$\begin{array}{l}\text { receive wind and } \\
\text { ingenerate lift force }\end{array}$} & \multirow{2}{*}{$\mathrm{F}[\mathrm{N}]$} & deformation & impossible to receive wind and \\
\hline & & & & & change in attack angle & ingenerate lift force \\
\hline \multirow{3}{*}{4.4} & \multirow{3}{*}{$\begin{array}{c}3.4 \text { (bolt), } \\
4.5 \text { (bearing) }\end{array}$} & \multirow{3}{*}{$\begin{array}{l}\text { principal } \\
\text { axis }(3.2)\end{array}$} & \multirow{3}{*}{$\begin{array}{c}\text { keep leveling rotation } \\
\text { part }\end{array}$} & \multirow{3}{*}{$\theta\left[{ }^{\circ}\right]$} & buckling & \\
\hline & & & & & bending deformation & impossible to keep leveling \\
\hline & & & & & 6.7 & \\
\hline \multirow{2}{*}{4.5} & \multirow{2}{*}{$\begin{array}{l}4.4 \text { (bearing), } \\
6.2 \text { (bolt) }\end{array}$} & \multirow{2}{*}{$\begin{array}{l}\text { rotation } \\
\text { part }(3.2)\end{array}$} & \multirow{2}{*}{$\begin{array}{l}\text { receive torque from } \\
\text { impeller }\end{array}$} & \multirow{2}{*}{$\omega[\mathrm{rad} / \mathrm{s}]$} & 6.6 & \multirow{2}{*}{$\begin{array}{l}\text { impossible to receive torque } \\
\text { from impeller }\end{array}$} \\
\hline & & & & & 6.7 & \\
\hline
\end{tabular}




\section{Case Study in Failure Modes Modeling for Micro Windmill}

The proposed process is applied to analyses of a microwindmill. The structure and the functional diagram of the micro windmill are shown in Figures 2 and 3, respectively. An undergraduate student of mechanical engineering has conducted the failure mode analyses and risk modeling. He had almost no knowledge about FMEA or risk assessment before participating in this study. Plus, he got no financial salary by participating in the study.

At first, he processed the failure analyses after taking the classes of FMEA and risk analyses and reading the traditional FMEA textbook. Subsequently the analysis by the proposed process was conducted by him. Each process took about 5 days to complete the FMEA worksheet. Figures 5 and 6 show the result of predicted failure modes by using both the process. The result of the proposed process yield more failure modes, cause of failures. Furthermore, the proposed process can determine more specified risk scenarios with failure modes.

The result strongly indicates that the proposed process can effectively support a practitioner with less knowledge or experiences in conducting FMEA and risk assessment. In production design, shortening the period of designs are critical to severely compete among global business environment. However, the shorter the design reviewing became, the more latent failure were missed which yields huge amount of recalls or losses. The current achievement will be helpful in training fresh engineers or improving the quality of design review processes as a proactive prevention technique. The current procedure in Figure 1 is to be improved in more logical identification process of failure modes from various types of abnormal modes in stress, strength and environmental factors. Even in the cases of veteran's case, the proposed process can be helpful by leading the veteran's points of view on inexperienced failure modes/risk scenario, which can reveal other failure modes in advance.

The proposed model will be effective if the management system, which include the data base of failure modes, design drawing, design review results etc. In order to apply the model, such the data base is necessary. The authors tested the effectiveness of web-based database system in design review management [8]. If the specific failure modes list is constructed, the proposed model can be applied not only the case study's product, but also the more normal products such as automobiles, manufacturing machines, robots etc. The specific failure modes list should be prepared in order to be selected with ease in their targeting products [5]. The authors have also conducted the effective list of failure modes.

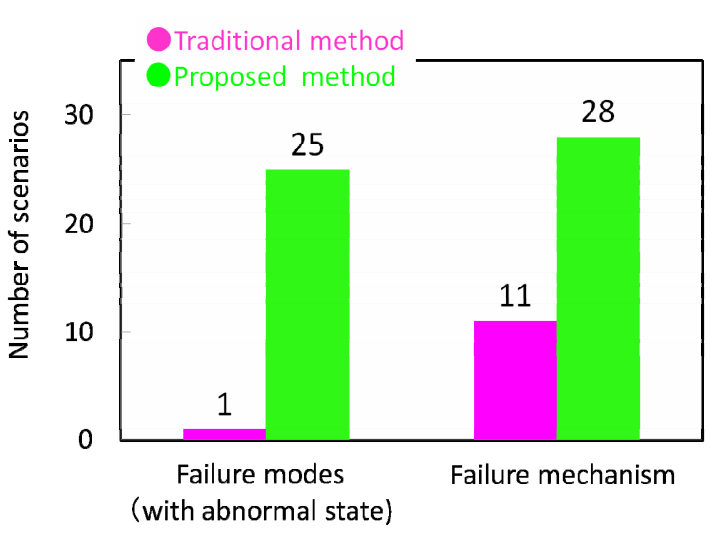

Figure 5. Comparison of FMEA result.

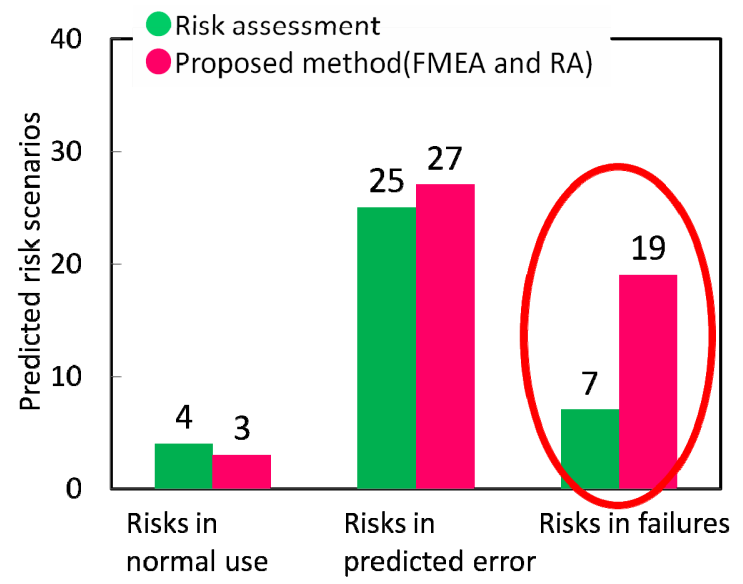

Figure 6. Comparison of risk assessment result.

\section{Conclusion}

The authors proposed the logical process of failure modes modeling by patterning the deviations from normal states of SSM (Stress-Strength Model). The case study certified the effectiveness of the proposed method in predicting more failure modes and risk scenarios with less experiences compared with a conventional process.

\section{Acknowledgements}

The author thanks to the partial support by JST program "Top-runner for industry-academy fusion incubation systems" at Nagaoka University of Technology and JST research project "Development of high pressure food processing vessel considering system safety".

\section{REFERENCES}

[1] M. G. Stewart and R. E. Melchers, "Probabilistic Risk Assessment of Engineering Systems," Morikita Publishing Inc., Tokyo, 2003, pp. 190-227.

[2] K. Suzuki, "Principle of Preventive Solutions and Its System," Nikkagiren Publishing, Tokyo, 2004, pp. 89-96. 
[3] T. Yoshimura, "Toyota-Style Preventive Solutions GD3," Nikkagiren Publishing, 2000, pp. 52-91.

[4] H. Shimizu and T. Yoshimura, "Reliability Problem Prevention Method of Stimulating Creativity Needed to Notice Problems," Transactions of the Japan Society of Mechanical Enginees Series C, Vol. 71, No. 706, 2004, pp. 230-237.

[5] H. Shimizu, Y. Otsuka and H. Noguchi, "Design Review Based on Failure Mode to Visualize Reliability Problems in the Development Stage of Mechanical Products," International Journal of Vehicle Design, Vol. 53, No. 3, 2009, pp. 149-165.

[6] Y. Otsuka, H. Shimizu and H. Noguchi, "Efficacious
Design Review Process Including on the Job Training in Finding Misunderstand Errors," International Journal of Reliability, Quality and Safety Engineering, Vol. 16, No. 3, 2009, pp. 281-302. doi:10.1142/S021853930900340X

[7] Y. Otsuka and H. Noguchi, "Noise-Adaptive System Modeling Procedure Based on the Heuristic Method Stimulated by Comparison," Transactions of the Japan Society of Mechanical Enginees Series C, Vol. 75, No. 761, 2010, pp. 207-216.

[8] Y. Otsuka, T. Yukawa and Y. Mutoh, "Development of Web-Based Design Review System for Reliability and Safety Knowledge Management," International Journal of Safety, Vol. 9, No. 2, 2010, pp. 22-28. 